\title{
Partial Anomalous Pulmonary Venous Return With Persistent Angina: Simulating Coronary Artery Disease
}

\author{
Vinod Namana ${ }^{\mathrm{a}, \mathrm{b}}$, Ram Balasubramanian ${ }^{\mathrm{a}}$, Sruthi Namana ${ }^{\mathrm{a}}$, Hollander Gerald ${ }^{\mathrm{a}}$
}

\begin{abstract}
Partial anomalous pulmonary venous return (PAPVR) is an uncommon adult congenital heart disease caused by an abnormal return of one or more, but not all, of the pulmonary veins to the right atrium or indirectly through venous connections from the anomalous pulmonary vein. Presentations vary from incidental findings to severe heart failure and diagnosis could easily be missed. We report a case of PAPVR in a 51-year-old male with a history of coronary artery bypass surgery presenting with exertional dyspnea and chest discomfort which were initially interpreted as angina in light of patient's known coronary disease and prior revascularization. Patient underwent surgical repair with redirection of the pulmonary connection to the left atrium. On follow-up, patient had symptomatic improvement and decrease in pulmonary artery pressures. Diagnosis of PAPVR may be particularly elusive when patient also has a more common diagnosis, such as coronary disease. Unexplained pulmonary hypertension should alert physicians to consider additional differential diagnoses including congenital heart disease despite the patient's adult age. As evidenced in this patient, successful surgical repair can lead to gratifying results and improved prognosis.
\end{abstract}

Keywords: Partial anomalous pulmonary venous connection; Congenital heart disease; Pulmonary hypertension; Angina; Exertional dyspnea; Heart failure; Left to right shunt; Anomalous pulmonary vein

\section{Introduction}

Partial anomalous pulmonary venous return (PAPVR) is a rare adult congenital heart disease caused by abnormal return of one or more, but not all, of the pulmonary veins to the right atrium (RA) or indirectly through venous connections from the

Manuscript accepted for publication September 22, 2015

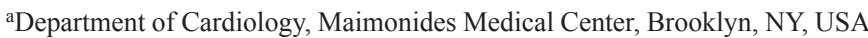
${ }^{b}$ Corresponding Author: Vinod Namana, Department of Cardiology, Maimonides Medical Center, 4802 10th Avenue, Brooklyn, NY 11219, USA.

Email:vnamana@gmail.com

doi: http://dx.doi.org/10.14740/jmc2309w anomalous pulmonary vein $[1,2]$. Presentations vary from incidental findings to severe heart failure [1] and diagnosis could easily be missed. Our patient with a history of coronary artery disease (CAD) presented with symptoms of exertional dyspnea and chest discomfort, simulating more common diagnosis of CAD, which eluded the diagnosis of PAPVR. Delay in diagnosis led to multiple emergency room visits, hospitalizations, coronary angiograms and numerous other investigations.

\section{Case Report}

A 51-year-old Asian male with a past medical history of CAD, type II diabetes mellitus, hypertension and dyslipidemia presented with persistent exertional dyspnea and chest discomfort. The patient underwent coronary artery bypass surgery at the age of 39, after he presented with progressive exertional angina and had a positive stress test for inducible ischemia in multivessel territories and coronary angiography showing severe triple vessel disease. During the past decade, despite medical optimization for CAD, he had persistent exertional symptoms of chest discomfort and dyspnea, which led to multiple emergency room visits and hospitalizations. These symptoms were interpreted as angina in light of the patient's known $\mathrm{CAD}$ and prior revascularization. His multiple coronary angiograms revealed patent grafts and stents, and gastroenterology workups which included esophagogastroduodenoscopy, abdominal ultrasonography and scintigraphic gastric emptying were normal. On admission, patient was hemodynamically stable and physical examination revealed jugular venous distension, loud P2 with wide, but not fixed, splitting of S2, holosystolic murmur at lower left sternal border and trace pedal edema. Electrocardiography showed incomplete right bundle branch block pattern. Chest radiography showed enlarged RA with prominent pulmonary vascular markings. Transthoracic echocardiogram showed enlarged RA and right ventricle (RV), severe pulmonary hypertension with pulmonary artery (PA) systolic pressure of $60 \mathrm{~mm} \mathrm{Hg}$ and normal left ventricular systolic function. Pulmonary hypertension was out of proportion to elevated left-sided pressure which prompted to do further evaluation. Patient was reluctant to undergo right heart catheterization but agreed to a transesophageal echocardiogram and a computed tomographic angiogram of chest (Fig. 1), which confirmed these findings and suggested the possibility 


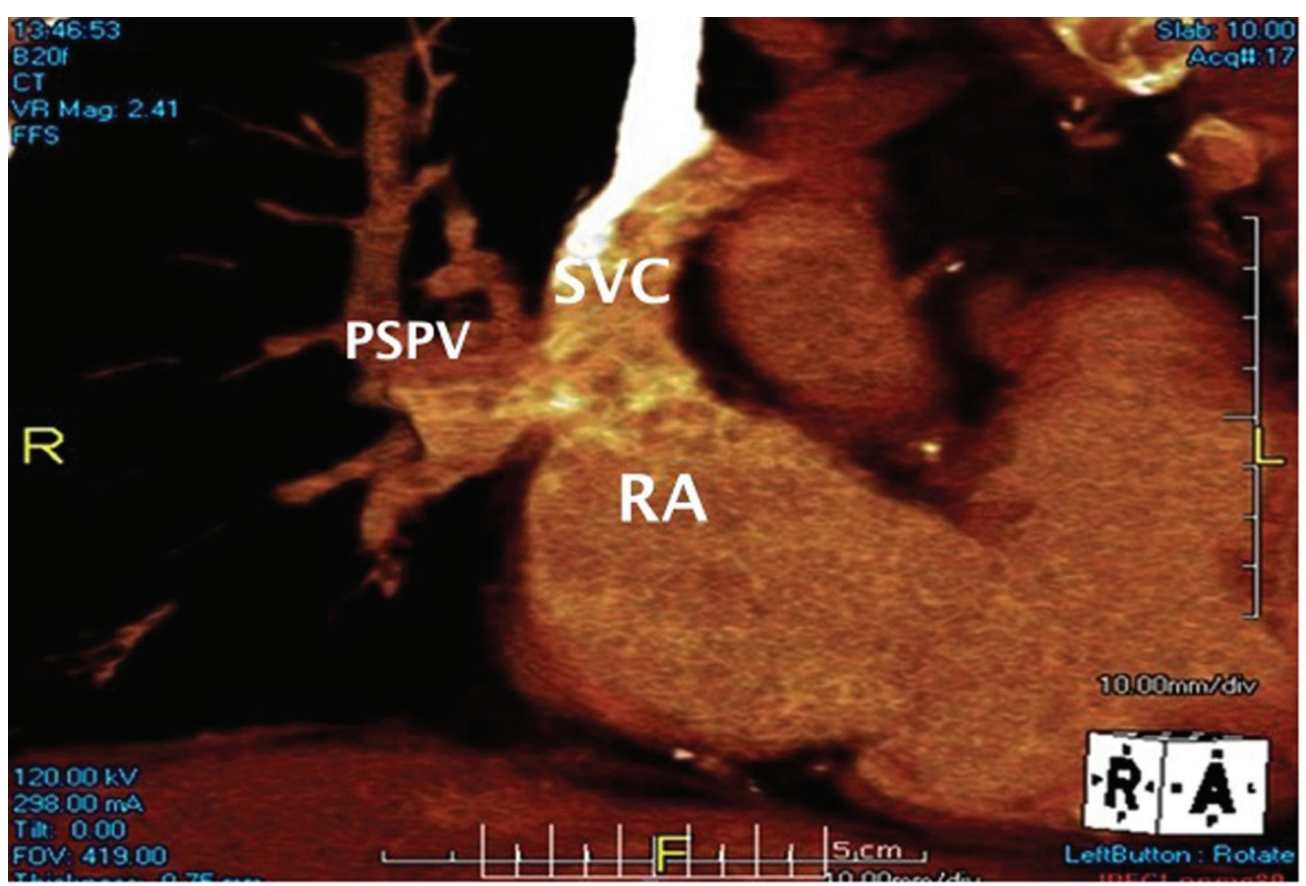

Figure 1. Computed tomography of the chest showing PSPV draining into RA and SVC. RA: right atrium; SVC: superior vena cava; PSPV: persistent superior pulmonary vein.

of anomalous pulmonary venous drainage of right superior pulmonary vein into RA. Patient underwent surgical repair with redirection of the pulmonary connection to the left atrium by creation of atrial septal defect and interatrial baffle with bovine pericardium. On follow-up, patient had symptomatic improvement as well as regression of RA, RV enlargement and a decrease in mean PA systolic pressure from $60 \mathrm{~mm} \mathrm{Hg}$ before surgery to $30 \mathrm{~mm} \mathrm{Hg} 2$ months after repair.

\section{Discussion}

PAPVR is uncommon venous anomaly occurring in incidence of $0.4-0.7 \%$ in antemortem and postmortem cases [1-3], but the real incidence would be higher given their silent nature of the disease and increased detection with highly sensitive imaging modalities such as computed tomography angiography (CTA), magnetic resonance angiography (MRA) and transesophageal echocardiography (TEE) [2, 4-7]. PAPVR develops when the right and left portion of pulmonary veins regress early while primitive pulmonary systemic connections are still present $[1,2,8]$. In $80-90 \%$ of cases, PAPVR arises from whole right lung or 1- 2 lobes of right lung draining most commonly to superior vena cava (SVC), and less commonly to RA, inferior vena cava (IVC), coronary sinus, azygous vein, portal vein or hepatic vein $[1,2,4,7,8]$. PAPVR arises from left lung drain to left brachiocephalic vein, innominate vein or coronary sinus $[1,2,8]$. PAPVR is usually associated with other congenital anomalies and occurrence of isolated PAPVR is very rare [1, 2]. Most commonly PAPVR is associated with high venosum or secundum type atrial septal defect (ASD) in $80 \%$ cases $[1,2$, $4,8]$ and followed by persistent left SVC and less commonly ventricular septal defects, pulmonary stenosis, mitral stenosis, etc. $[1,2,9]$.

PAPVR is uncommon in pediatric age group and very rarely seen in adults $[1,2,4]$. Occurrence is twice as common in males $[1,8]$. Presentation varies from incidental findings to dyspnea, chest discomfort on exertion or at rest to severe heart failure and could present until fourth to seventh decade in life $[1,2]$. PAPVR acts as left to right shunt, emptying oxygenated blood into right heart giving rise to step up in oxygenation and in over time leading to pulmonary hypertension (PAH), RA, $\mathrm{RV}$ overload and right heart failure (RHF) from increased pulmonary resistance $[2,9]$. Our patient presented with persistent exertional dyspnea and chest discomfort, which was secondary to PAH, and RHF but misinterpreted his symptoms to CAD, which is a most common diagnosis.

In past, PAPVR is diagnosed with cardiac catheterization and cardioangiography $[1,4]$ and confirmed during surgery [1]. Chest radiography and TTE lack sensitivity [2] and the newer modalities of imaging such as CTA, MRA, and TEE are highly sensitive and specific in detection of PAPVR [2, 7]. Cardiac catheter based angiography and TTE do not detect PAPVR when there is no clinical suspicion or ASD [7]. Our patient had multiple cardiac catheter based angiography and TTE but did not detect PAPVR as there was no clinical suspicion and ASD. TTE showed RA, RV enlargement and severe PAH, which were confirmed by TEE. PAPVR was detected on TEE and confirmed by CTA. Recently noninvasive cardiac MRI/MRA without ionizing radiation is being used to define structural abnormalities and quantification of shunt volume $(\mathrm{Qp} / \mathrm{Qs})$ - flow through the pulmonary arterial bed to systemic arterial bed $[2,7]$.

General principle in treatment is to decrease the pulmo- 
nary vascular resistance and right heart overload. Asymptomatic patients without PAH need no intervention but need close monitoring for signs of PAH $[2,9]$. Symptomatic patients with $\mathrm{PAH}$ and Qp/Qs shunt ratio greater than 1.5 - 2 need surgery $[1,2,9]$ and if not a candidate for surgery, medical therapy and catheter embolization of anomalous vein play a pivotal role [2, 9]. A surgery is safe, effective in symptom and shunts reversal [2] and is associated with less than $1 \%$ mortality $[1,2]$. Our patient underwent surgical repair and resulted in improvement of symptoms, reversal of shunt, decrease in PAH, RA, and RV size. Patients with severe PAH or Eisenmenger's physiology need lung or heart lung transplant $[2,9]$.

\section{Conclusion}

The diagnosis of PAPVR may be particularly elusive when the patient also has a more common diagnosis, such as CAD. Unexplained pulmonary hypertension and enlarged right-sided chambers should alert physicians to consider additional differential diagnoses including congenital heart disease despite the patient's adult age. As evidenced in this patient, successful surgical repair can lead to gratifying results and improved prognosis.

\section{Grant Support}

None.

\section{Conflicts of Interest}

None.

\section{References}

1. Senocak F, Ozme S, Bilgic A, Ozkutlu S, Ozer S, Saraclar M. Partial anomalous pulmonary venous return. Evaluation of 51 cases. Jpn Heart J. 1994;35(1):43-50.

2. Sears EH, Aliotta JM, Klinger JR. Partial anomalous pulmonary venous return presenting with adult-onset pulmonary hypertension. Pulm Circ. 2012;2(2):250-255.

3. Healey JE, Jr. An anatomic survey of anomalous pulmonary veins: their clinical significance. J Thorac Surg. 1952;23(5):433-444.

4. Ammash NM, Seward JB, Warnes CA, Connolly HM, O'Leary PW, Danielson GK. Partial anomalous pulmonary venous connection: diagnosis by transesophageal echocardiography. J Am Coll Cardiol. 1997;29(6):13511358.

5. Haramati LB, Moche IE, Rivera VT, Patel PV, Heyneman L, McAdams HP, Issenberg HJ, et al. Computed tomography of partial anomalous pulmonary venous connection in adults. J Comput Assist Tomogr. 2003;27(5):743-749.

6. Ho ML, Bhalla S, Bierhals A, Gutierrez F. MDCT of partial anomalous pulmonary venous return (PAPVR) in adults. J Thorac Imaging. 2009;24(2):89-95.

7. Prasad SK, Soukias N, Hornung T, Khan M, Pennell DJ, Gatzoulis MA, Mohiaddin RH. Role of magnetic resonance angiography in the diagnosis of major aortopulmonary collateral arteries and partial anomalous pulmonary venous drainage. Circulation. 2004;109(2):207-214.

8. Snellen HA, van Ingen HC, Hoefsmit EC. Patterns of anomalous pulmonary venous drainage. Circulation. 1968;38(1):45-63.

9. Diller GP, Gatzoulis MA. Pulmonary vascular disease in adults with congenital heart disease. Circulation. 2007;115(8):1039-1050. 